\title{
Gelation in free-radical crosslinking copolymerization; fast transient fluorescence study
}

\author{
Ö. Pekcan and D. Kaya \\ Istanbul Technical University, Department of Physics, Maslak, 80626 Istanbul, Turkey
}

\begin{abstract}
The fast transient fluorescence (FTRF) technique was used to study the sol-gel phase transition in free-radical crosslinking copolymerization (FCC) in two different monomeric systems. Pyrene $\left(\mathrm{P}_{\mathrm{y}}\right)$ was used as a fluorescence probe for the in situ polymerization experiments. The fluorescence lifetimes of Py from its decay traces were measured and used to monitor the gelation process. Monomer consumption profiles were determined during gelation process using Stern-Volmer model. Gelations were performed at various temperatures and in various crosslinker contents. Composite rate constants, $k_{r}$ were determined and gelation activation energies, $\Delta E_{G}$ were measured.
\end{abstract}

\section{INTRODUCTION}

Because of its long excited singlet lifetime, pyrene $\left(\mathrm{P}_{\mathrm{y}}\right)$ as a chromophore [1] is an attractive choice for studying dynamics in polymers. $\mathrm{P}_{\mathrm{y}}$ has been successfully employed as the fluorescence probe in the study of micellar [2] and phospholipid dispersion [3]. These studies focus on the use of dynamics of quenching of $\mathrm{P}_{\mathrm{y}}$ monomer fluorescence and excimer formation processes. The other application of the use of $\mathrm{P}_{\mathrm{y}}$ as a fluorescence probe is the study of the vibronic fine structure of its monomer fluorescence. The intensities of the various vibronic bands show a strong dependent on the solvent environment [4]. In the presence of polar solvents there is an enhancement in the intensity of the 0-0 band whereas there is a little effect on other bands, thus the ratio of intensities of these bands has been used to study environmental change. For about two decades the transient fluorescence (TRF) technique for measuring fluorescence decay has been routinely applied to study many polymeric systems using dyes both as a probe and/or as labels [5-9]. TRF spectroscopy with direct energy transfer (DET) and quenching method has been used to characterize internal morphologies of composite polymeric materials $[10,11]$. Quenching besides DET is the general word used to describe any bimolecular process, which decreases the emission decay rate. The most important feature of these quenching mechanisms involves interactions between groups over different interaction distances. A single-photon counting (SPC) technique which produces decay curves and measures lifetimes in conjuction with DET was used to study the diffusion of small dye molecules within the interphase domain of anthracene and/or phenanthrene labelled poly (methyl methacrylate) (PMMA) particles [12].

In chemical gelation, the molecules crosslink into larger clusters by forming covalent bonds in various ways. In condensation polymerization, a network is formed by polymerizing bifunctional units and polyfunctional units serve as crosslinkers. Free-radical crosslinking coplymerization (FCC) has also been widely used to synthesize polymer gels. Several theories have been developed in the past half-century to describe gel formation in FCC, among which percolation theory provides a basis for modelling sol-gel phase transition [13-15]. The percolation models based on simulation in n-dimensional space can predict critical exponents or gel fraction, weight-average degree of polymerization, radius of gyration, etc. near the sol-gel phase transition called the critical region. This theory is, however, unrealistic outside of this region due to the difficulty of introduction of realistic mobilities. Another type of theories called mean-field theories such as the statistical and kinetic theories bases on a "tree approximation". Statistical theories originate from Flory [16] and Stockmayer [17] and assume equal reactivities of functional groups and the absence of cyclization reactions. The critical exponents in percolation theory differ from those found in Flory-Stockmayer. In FCC, the formation of bonds building the network can be described using differential equations with reaction time or monomer conversion as the independent variable. The kinetic approaches can take into account all the kinetic features of copolymerization and crosslinking reactions, which may suggest a more realistic approach to the mechanism of gelation, process [18-21]. Kinetic models have been extensively used to describe the relations among the molecular weight of polymer and the conversion or reaction time during crosslinking process. In the classical kinetic theory, the rate constant is proportional to the product of the number functional groups in each reactant. Modification of the classical kinetic theory by using rate constant that also depend on the structural features of the reactants has been done [22].

TRF and steady-state fluorescence (SSF) techniques were employed to study isotactic polystyrene in its 
gel state. A Pyrene derivative was used as a fluorescence molecule to monitor the polymerization, aging and drying of aluminosilicate gels [23]. These results were interpreted in terms of the chemical changes occurring during the sol-gel process and the interactions between the chromophores and the sol-gel matrix. We reported in situ observations of the sol-gel phase transition in free-radical crosslinking copolymerization using the SSF technique [24-30]. The bond percolation model was employed to obtain some critical exponents during sol-gel transitions of such a system.

In this work, the strobe technique, which is named as fast transient fluorescence (FTRF), was used to study the sol-gel transition in FCC of methyl methacrylate (MMA) with ethylene glycol dimethacrylate (EGDM) and styrene (S) with divinylbenzene (DVB) systems respectively. The major advantage of the strobe technique over other lifetime instruments is the time duration of a single experiment, which takes only seconds. In this work, this advantage of strobe technique was employed to make at least 20 to 30 lifetime measurements during the gelation of MMA with EGDM and S with DVB over a time interval of one hour. In-situ, FTRF experiments were carried out by illuminating the sample cell during gelation, and fluorescence decay traces were observed using Strobe Master System (SMS). The SternVolmer quenching equation was employed to monitor MMA and/or S consumptions during the sol-gel phase transition, from which composite rate constants, $k_{r}$ were determined and used to measure gelation activation energies.

\section{FLUORESCENCE QUENCHING}

Fluorescence intensities of aromatic molecules are affected by both radiative and non-radiative processes [31]. If the possibility of perturbation due to oxygen is excluded, the radiative probabilities are found to be relatively independent of environment and even of molecular species. Environmental effects on nonradiative transitions that are primarily intramolecular in nature are believed to arise from a breakdown of the Born-Oppenheimer aproximation [32]. The role of the solvent in such a picture is to add the quasi-continuum of states needed to satisfy energy resonance conditions. The solvent acts as an energy sink for rapid vibrational relaxation that occurs after the rate limiting transition from the initial state. Years ago, Birks et al. studied the influence of solvent viscosity on fluorescence characteristics of pyrene solutions in various solvents and observed that the rate of monomer internal quenching is affected by solvent quality [33]. Weber et al. reported the solvent dependence of energy trapping in phenanthrene block polymers and explained the decrease in fluorescence as due to the static quenching, caused by the solvent induced trapping states [34]. A matrix that changes little with temperature will enable one to study molecular properties themselves without changing environmental influence. Poly (methly methacrylate) (PMMA) has been used as such a matrix in many studies [35].

Excitation of ground state dye $F$ to its singlet excited state $F^{*}$ given

$$
F+h v \longrightarrow F^{*}
$$

Emission of the fluorescence is the radiative transition of an electronically excited molecule from its singlet excited state to its ground state [1]

$$
F^{*} \longrightarrow F+h v^{\prime} .
$$

Fluorescence quenching normally refers to any bimolecular process between the excited singlet state of a fluorescence dye and the second species that enhances the decay rate of the excited state. One can schematically represent the process as

$$
F^{*}+Q \frac{k_{1}}{k_{-1}}\left(F^{*} Q\right) \stackrel{k_{2}}{\longrightarrow} F+Q,
$$

where $F$ and $F^{*}$ represents the fluorescent molecule, and its excited form, $\mathrm{Q}$ is the quencher. $k_{1}, k_{-1}$ and $k_{2}$ represent the diffusional, and internal quenching rate constants respectively. Many types of processes lead to quenching. Kinetically, quenching processes can be divided into two main categories; dynamic and static. In dynamic quenching, diffusion to form an encounter pair $\left(F^{*} Q\right)$ during the excited state lifetime of the dye leads to quenching. In static quenching, diffusion does not occur (and hence is out side our interest here). Dynamic quenching is most likely to occur in fluid solution, where the dye or quencher is free to move.

Here the quenching efficiency is given by

$$
\gamma=\frac{k_{2}}{k_{-1}+k_{2}+\tau_{0}^{-1}+k_{1}[Q]},
$$

where $\tau_{0}$ is the lifetime of $F^{*}$ where no quenching takes place and $[Q]$ represents the quencher concentration. If $k_{2} \gg\left(k_{-1}+\tau_{0}{ }^{-1}+k_{1}[Q]\right)$ then equation (1) produces $\gamma \approx 1$ and $k_{1}=k_{q}$ Here $k_{q}$ is the quenching rate constant and given by Smoluchowski relation[1]. The rate equation for $\left[F^{*}\right]$ with pulse excitation can be written as

$$
\frac{d\left\lfloor F^{*}\right\rfloor}{d t}=-\tau^{-1}\left[F^{*}\right]+L\left(t-t^{\prime}\right)[F],
$$

where $\left[F^{*}\right]$ and $[F]$ represents the concentration of $F^{*}$ and $F$ molecules and $L\left(t-t^{\prime}\right)$ is the light pulse of SMS system. Here $\tau^{-1}$ is given by the following relation call Stern-Volmer equation

$$
\tau^{-1}=\tau_{0}^{-1}+k_{q}[Q] .
$$


Solution of equation (2) produces the decaying fluorescence intensity as

$$
I(t)=A \exp \left[-\frac{t}{\tau}\right]
$$

where $A$ is the preexponential factor. The observed fluorescence decay of a sample, $\phi(t)$ is related to the actual fluorescence decay, $I(t)$ and the SMS light pulse by the convolution integral

$$
\phi(t)=\int_{0}^{t} L\left(t-t^{\prime}\right) I\left(t^{\prime}\right) d t^{\prime} .
$$

\section{KINETIC MODEL}

The first step in free-radical polymerization is the decomposition of the initiator molecule with the rate constant, $k_{i}$ into two species carrying unpaired electrons called free radicals. A free radical can then react to open the double bond of a vinyl monomer and add to it, with one electron remaining unpaired. In a very short time, usually a few seconds or less, many more monomers add successively to the growing chain with the propagation rate constant, $k_{p}$. Finally two radicals react to end each other's growth activity and form one or more polymer molecules [36]. This bimolecular process is called termination reaction and is identified with the rate constant, $k_{t}$. During the free-radical crosslinking copolymerization (FCC), addition of divinyl monomers to the growing chain results in the formation of polymer molecules with reactive sites ("pendant vinyl groups"). These reactive sites on polymer chains offer the possibility of forming chemical structures of macroscopic dimensions called polymer gels. The rate of consumption of monomer is usually called the rate of polymerization and is given by the following equation

$$
\frac{d[M]}{d t}=-\frac{k_{p} k_{i}^{1 / 2}}{k_{t}^{1 / 2}}[M][I]^{1 / 2}
$$

which is applied only under steady-state conditions. Here $[M]$ and $[I]$ are the concentration of monomer and initiator respectively. Often the rate constant for initiation, $k_{i}$ is large and only a small proportion of the initiator breaks down into radicals, which means that [I] stays constant during polymerization process and equation (6) can be written as

$$
\frac{d[M]}{d t}=-k_{r}[M]
$$

where $k_{r}$ is the composite rate constant. The solution of the equation (7) produces the relation for the monomer consumption as follows

$$
[M]=\left[M_{0}\right] \exp \left(-k_{r} t\right)
$$

where $\left[M_{0}\right]$ is the concentration of monomer at $t=0$.

\section{EXPERIMENTAL}

4.1. Materials. In this work we monitored the gelation in FCC of methyl methacrylate (MMA) with ethylene glycol dimethacrylate (EGDM) and styrene (S) with divinylbenzene (DVB) by using the in situ FTRF technique. EGDM and DVB have been commonly used as crosslinker in the synthesis of polymeric networks. Here, for our use, the monomers MMA (Merck) and EGDM (Merck) were freed from the inhibitor by shaking with a $5 \%$ aqueous $\mathrm{NaOH}$ solution, washing with water and drying over sodium sulfate. They were then distilled under reduced pressure over copper chloride. The initiator, AIBN (Merck), was recrystallized twice from methanol. Here EGDM (0.015\%) and AIBN (0.26 wt\%) was dissolved in MMA and this solution was transferred into round quartz cell of $10 \mathrm{~mm}$ internal diameter for the fluorescence decay measurements.

Styrene (S, Merck) and commercial DVB (DVB, Merck) were also shaken with $5 \% \mathrm{NaOH}$, washed with water, dried with anhydrous $\mathrm{CaCl}_{2}$, and finally distilled under reduced pressure. Purity was checked by gas chromatography. Various batches of DVB solution were used. Batch analyses ranged between $50-60 \%$ DVB isomers (m-DVB : p-DVB ratio $=3-3.2: 1$ ), the rest being ethylstyrene. Benzoyl peroxide (BP, Merck) was recrystallized from acetone solution.

4.2. Gelation. The radical copolymerization of MMA with EGDM was performed in bulk in the presence of 2,2'-azobisisobutyronitrile (AIBN) as an initiator at four different temperatures $\left(50,55,60\right.$ and $65^{\circ} \mathrm{C}$ ). $\mathrm{P}_{\mathrm{y}}$ lifetimes were measured to detect the gelation process where MMA act as an energy sink for the excited $\mathrm{P}_{\mathrm{y}}$ molecules. The PMMA network however provides an ideal, unchanged environment for the excited $\mathrm{P}_{\mathrm{y}}$ molecules. Naturally, from these experiments one may expect a drastic increase in $\mathrm{P}_{\mathrm{y}}$ lifetimes during gelation process.

The free-radical crosslinking copolymerization (FCC) of S with DVB was also performed in bulk in the presence of $\mathrm{BP}$ as an initiator. The initiator concentration was held constant at $[I]_{0}=0.100 \mathrm{M}$. The reaction mixtures were prepared by dissolving BP in the selected S - DVB monomer mixture and flushing with nitrogen 10 min prior to polymerization. In this work, different DVB content in the range from 0.025 to $0.20 \mathrm{vol} \%$ was used for each FCC experiment at a constant temperature $\left(70{ }^{\circ} \mathrm{C}\right)$.

4.3. Fluorescence measurements. in situ fluorescence decay experiments from which $\mathrm{P}_{\mathrm{y}}$ lifetimes can be determined were performed using Photon Technology International's (PTI) Strobe Master System (SMS). In the strobe, or pulse sampling technique [37] the sample is excited with a pulse light source. The name comes about because the PMT is gated or strobed by a voltage pulse that is synchronized with the pulsed light source. 
The intensity of fluorescence emission is measured in a very narrow time window on each pulse and saved in a computer. The time window is moved after each pulse. The strobe has the effect of turning of the PMT and measuring the emission intensity over a very short time window. When the data has been sampled over the appropriate range of time, a decay curve of fluorescence intensity versus time can be constructed. Because the strobe technique is intensity-dependent, the strobe instrument is much faster than Single Photon Counting (SPC) and even faster than a phase instrument. The strobe instrument is much simpler to use than SPC and data is easier to interpret than with a phase system. Because of these advantages SMS is used to monitor gelation processes which take around few hours.

All lifetime measurements were made at $90^{\circ}$ to the incident beam and the slit widths were kept at $10 \mathrm{~nm}$. The gelation experiment was performed in a round quartz cell, which was placed in the SMS, and fluorescence decays were collected over three decades of decay time. The sample was illuminated with $350 \mathrm{~nm}$ excitation light and pyrene fluorescence emission was detected at $393 \mathrm{~nm}$. Deconvolution of $I(t)$ from equation (5) is performed using iterative linearleast-squared fitting technique. The uniqueness of the fit of the data to the model is determined by $x^{2}\left(x^{2}<\right.$ $1.20)$, the distribution of weighted residuals, and the autocorrelation of the residuals. All measurements were made at $70{ }^{\circ} \mathrm{C}$ temperature and sample was deoxygenated by bubbling nitrogen through for 10 minutes.

\section{RESULTS AND DISCUSSION}

5.1. Gelation of MMA at various temperatures. Figure 1 presents the fluorescence decay profiles of $\mathrm{P}_{\mathrm{y}}$ in MMA at various gelation steps. It is observed that as the gelation time, $t_{g}$ is increased, excited pyrenes decay slower and slower by indicating that quenching of excited pyrenes decrease. Here the gelation time, $t_{g}$ is defined as the elapsed time during gelation process. In order to monitor gelation processes, the fluorescence decay curves were measured and were fitted to the following equation.

$$
I=I_{0} \exp \left(\frac{-t}{\tau}\right)
$$

where $I$ and $I_{0}$ are the intensity of $\mathrm{P}_{\mathrm{y}}$ at time $t$ and zero and $\tau$ is the lifetime of $\mathrm{P}_{\mathrm{y}}$. A typical decay curve and its fit to equation (9) is shown in Figure 2. $\tau$ values were produced at each gelation step using linear least squares analysis and are plotted versus $t_{g}$ in Figure 3. In order to quantify these results, Stern-Volmer type of quenching mechanism is proposed for the fluorescence decay of $\mathrm{P}_{\mathrm{y}}$ during gelation process, where equation (3) can be employed and rewritten as follows

$$
\tau^{-1}=\tau_{0}^{-1}+k_{q}[\mathrm{MMA}]
$$

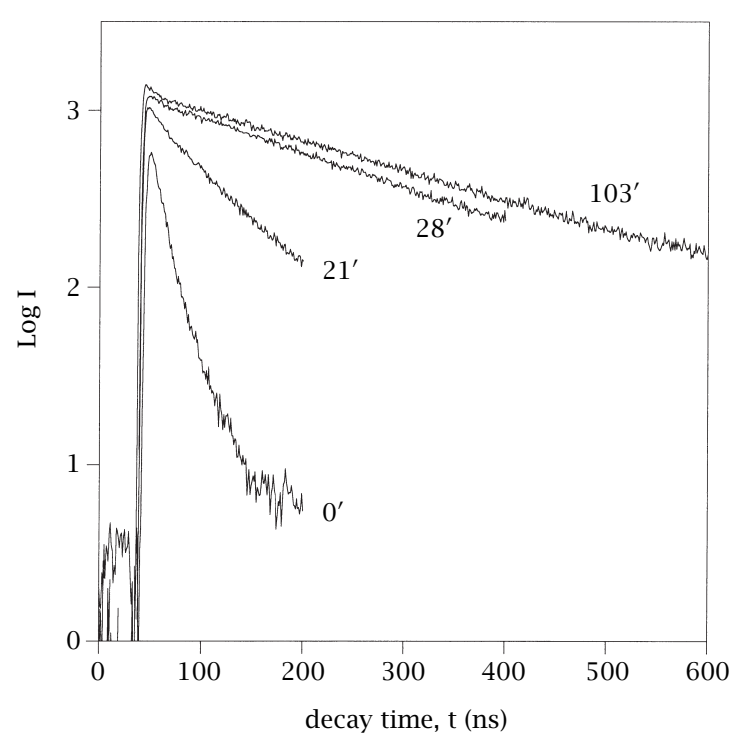

Figure 1. Fluorescence decay profiles of excited $\mathrm{P}_{\mathrm{y}}$, at various gelation steps. I is the $\mathrm{P}_{\mathrm{y}}$ intensity. Numbers on each curve present the gelation times in minute.
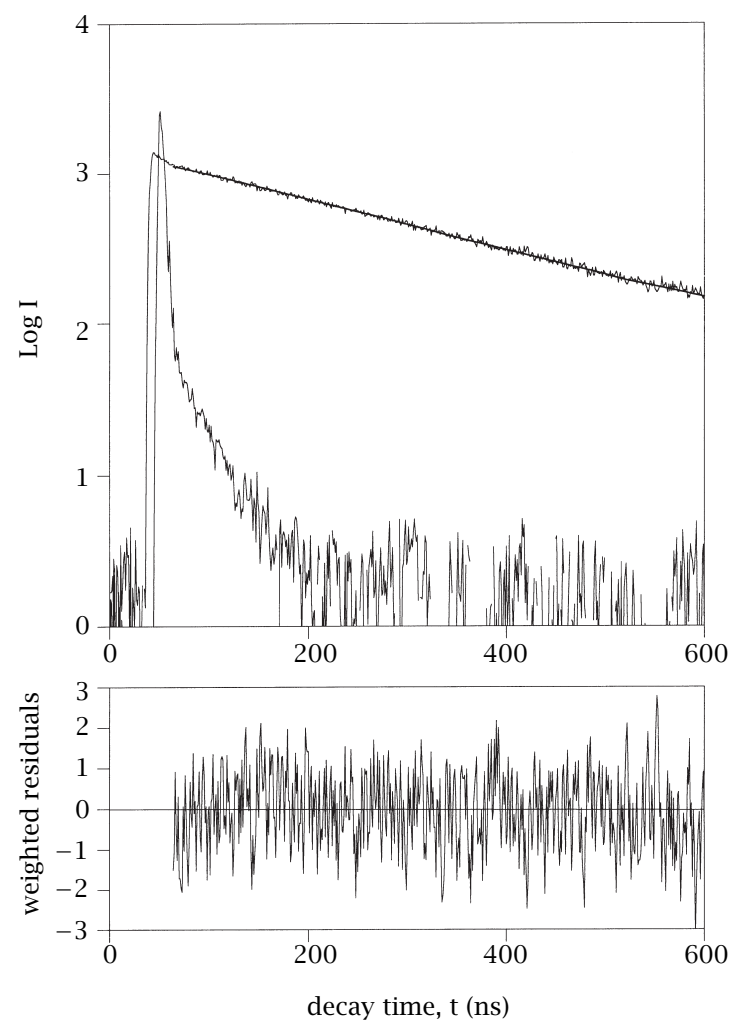

Figure 2. The fit of the decay curve of $\mathrm{P}_{\mathrm{y}}$ according to equation (9), at the gelation time of $103 \mathrm{~min}$. The sharp peaked curve is the lamp profile.

Here it is assumed that MMA is the only quencher for the excited $\mathrm{P}_{\mathrm{y}}$ molecules. $\tau_{0}$ was taken as $200 \mathrm{~ns}$ at $t_{g}$ of $80 \mathrm{~min}$, where the gelation is completed and the 


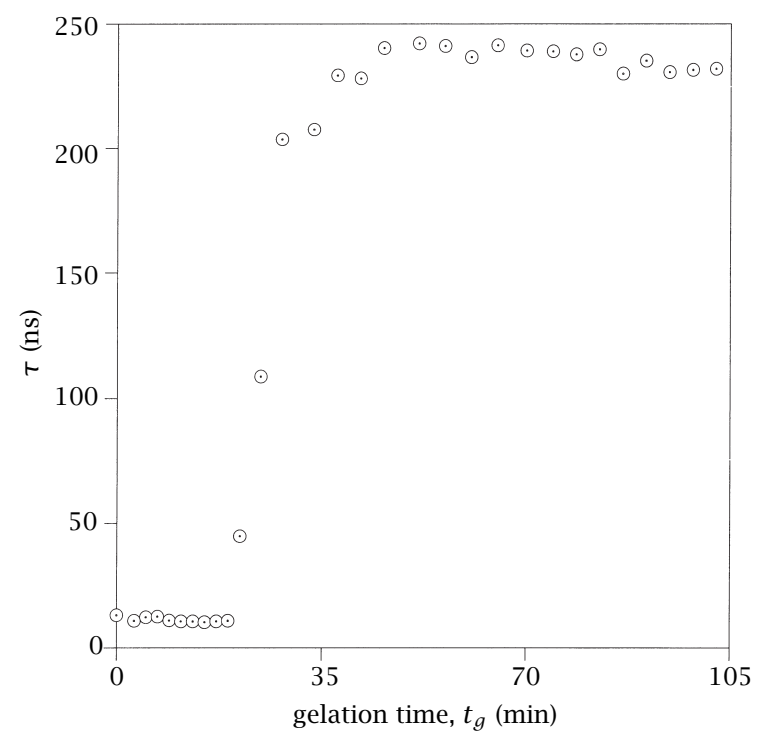

Figure 3. Plot of the $\mathrm{P}_{\mathrm{y}}$ lifetimes, $\tau$ versus gelation time, $t_{g}$.

ideal solid network is reached. $k_{q}$ was measured before the polymerization process is started and found to be $9.14 \times 10^{6} \mathrm{M}^{-1} \mathrm{~s}^{-1}$, where $\left[\mathrm{MMA}_{0}\right]$ was taken as $9.4 \mathrm{M}$. Using $k_{q}$ value and the measured $\tau$ values, [MMA] values were obtained from equation (10) and are plotted versus effective gelation time $\left(t_{g}-t_{0}\right)$ in Figure 4 , where $t_{0}(=20 \mathrm{~min})$ was chosen as the onset of gelation time which corresponds to the $\left[\mathrm{MMA}_{0}\right]$ value. As seen in Figure 4 the consumption curve of [MMA] obeys the exponential relation in equation (8). The fit of the data in Figure 4 to equation (8) is given in Figure 5 where the slope of the linear relation produced the composite rate constant, $k_{r}$ as $9 \times 10^{-3} \mathrm{~s}^{-1}$ for the polymerization during gelation.

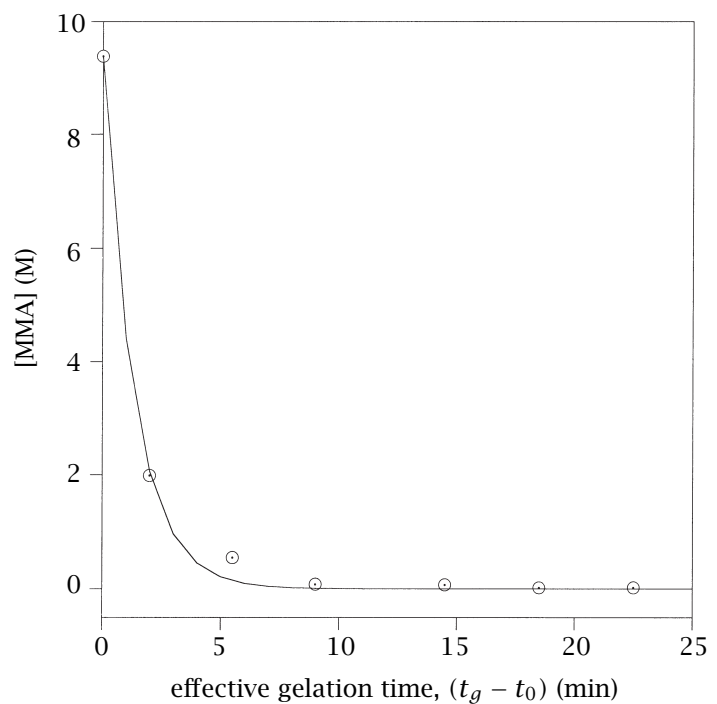

Figure 4. Plot of the monomer, consumption versus $\left(t_{g}-t_{0}\right)$ during gelation where $t_{0}=20 \mathrm{~min}$ is the time for the onset of gelation.

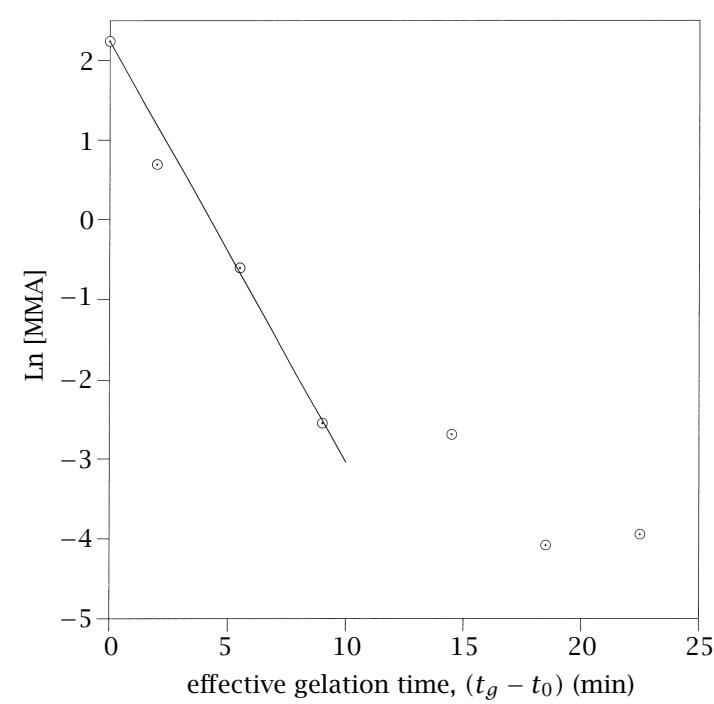

Figure 5. The fit of the data in Figure 4 to equation (8). The slope of the linear relation produce the composite rate constant, $k_{r}$ for the polymerization of MMA.

$\tau$ values obtained during FCC are plotted versus gelation time, $t_{g}$, in Figure 6(a), (b) and (c) for 50, 55
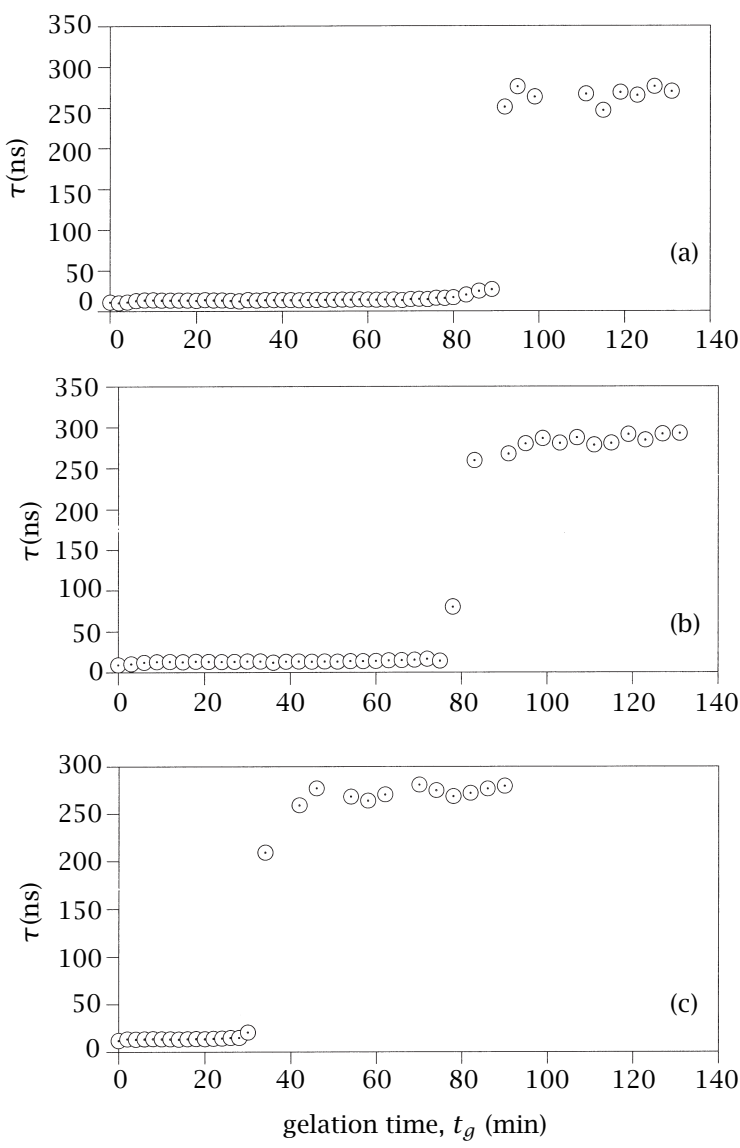

Figure 6. Plot of the $\mathrm{P}_{\mathrm{y}}$ lifetimes, $\tau$ versus gelation time, $t_{g}$ for the gelation temperatures of $a-50, b-55$ and $c-60^{\circ} \mathrm{C}$. 
and $60{ }^{\circ} \mathrm{C}$ temperature samples, respectively. The produced [MMA] consumption curves versus $t_{g}$ obey the relation in equation (8). The slope of the linear relations in Figure 6 produce the composite rate constants, $k_{r}$ according to equation (8), for polymerizations during gelation at various temperatures. It is observed that, as the temperature is raised composite rate constant increase as expected. A simple Arrhenius treatment to this data in Figure 7(a) produces the gelation activation energy, $\Delta E_{G}$ as $15.6 \mathrm{kcalmol}^{-1}$ for this given temperature range. Here in the following relation was used

$$
k_{r}=k_{r^{0}} \exp \left(-\frac{\Delta E_{G}}{k T}\right)
$$

where $k_{r^{0}}$ is the composite rate constant at infinite temperature, and $k$ is the Boltzman constant.

Similar Arrhenious treatment can also be done for the onset of gelation time $t_{0}$ as

$$
t_{0}^{-1}=t_{00}^{-1} \exp \left(-\frac{\Delta E_{I}}{k T}\right)
$$

where $t_{00}$ is the $t_{0}$ at infinite temperature and $\Delta E_{I}$ is the gelation activation energy. In Figure 7(b), logarithmic plot of $t_{0}^{-1}$ versus inverse temperature is presented where the slope of the linear relation produced the $\Delta E_{I}$ value as $15.5 \mathrm{kcal} \mathrm{mol}^{-1}$ which is very close to the $\Delta E_{G}$ value. From here one may conclude that both rate
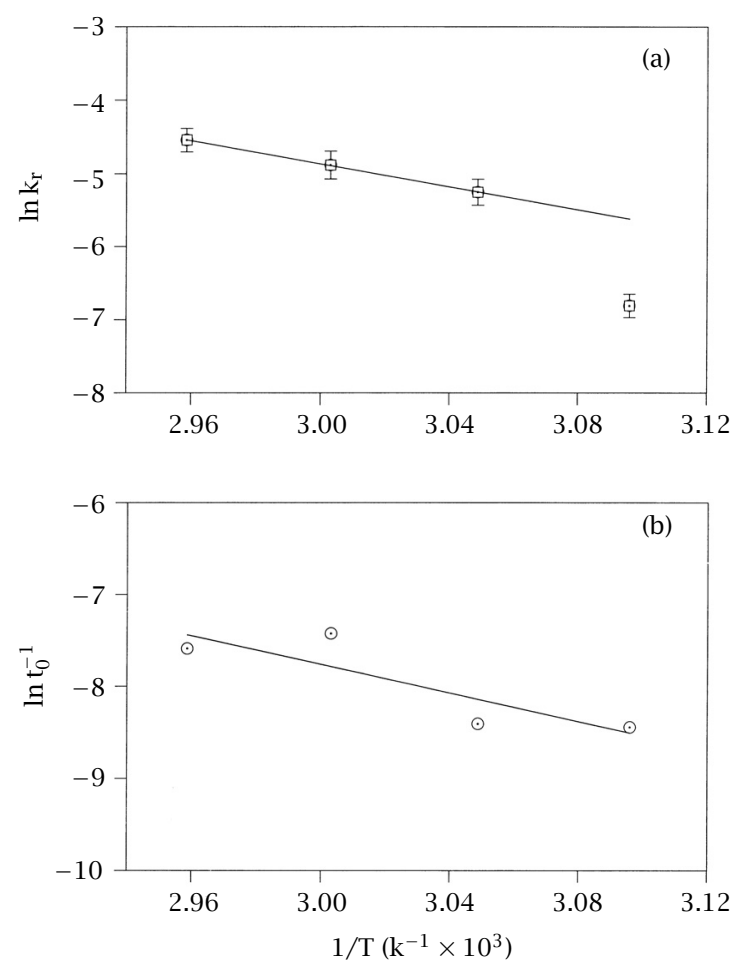

Figure 7. Logarithmic plots of a-composite rate constant, $k_{r}$ and $b-t_{0}^{-1}$ versus inverse temperature, $T$ according to Arrhenious relations. equations (11) and (12) produces the similar activation energies which are also very close to the our early finding for FCC process during bulk polymerization of MMA.

5.2. Gelation of $S$ in various crosslinker contents. In order to monitor gelation processes in S with DVB system, the fluorescence decay curves were also measured and then fitted to equation (9). $\tau$ values obtained during FCC are plotted versus gelation time, $t_{g}$, in Figure 8(a), (b) and (c) for 0.025, 0.05 and 0.1\% DVB content samples, respectively. As seen in Figure 8, Py lifetimes, $\tau$ increased drastically from very low values $(3 \mathrm{~ns})$ to their unquenched $\left(\tau_{0}\right)$ values during gelation at each DVB content sample. The onset of gelation time $\left(t_{0}\right)$ at which sol-gel transition starts, decreased as DVB content is increased, indicating the early gelation process takes place at high DVB content. Figure 9 represents the plot of $t_{0}$ versus DVB. In order to quantify the behaviour of $\tau$ versus time, the Stern-Volmer type of quenching mechanism is proposed for the fluorescence decay of $\mathrm{P}_{\mathrm{y}}$ during gelation process, where equation (3) can be employed and rewritten as follows

$$
\tau^{-1}=\tau_{0}^{-1}+k_{q}[\mathrm{~S}] .
$$
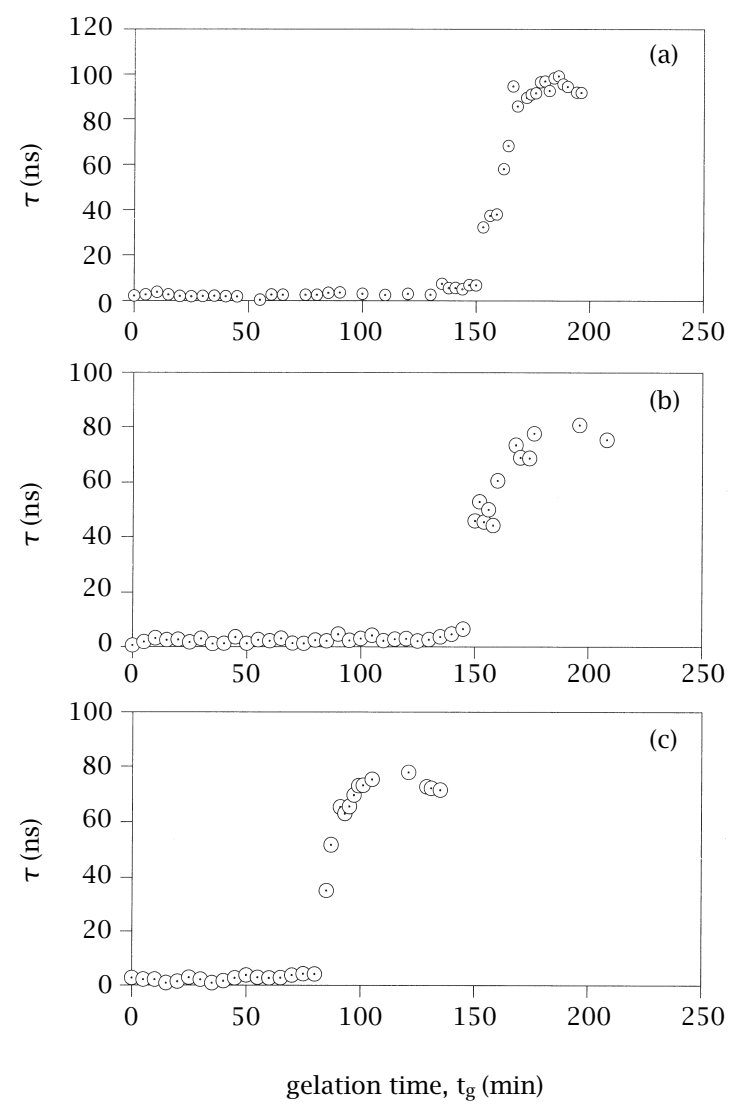

Figure 8. Plot of the $\mathrm{P}_{\mathrm{y}}$ lifetimes, $\tau$ versus gelation time, $t_{g}$, for $a-0.025, b-0.05$ and $c-0.1 \% D V B$ content samples. 


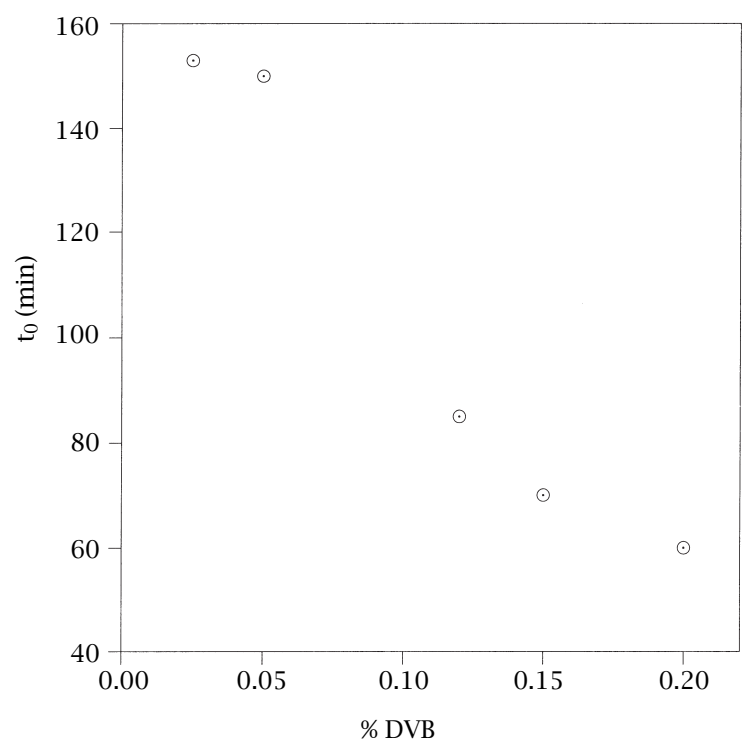

Figure 9. Plot of the onset of gelation, $t_{0}$, versus crosslinker contents.

Here it is assumed that $\mathrm{S}$ is the only quencher for the excited $\mathrm{P}_{\mathrm{y}}^{*}$ molecules, i.e. most of the bimolecular quenching processes take place between $\mathrm{S}$ and $\mathrm{P}_{\mathrm{y}}^{*}$, because the amount of DVB in the system is negligible compared to $\mathrm{S}$ molecules. $\tau_{0}$ values between $100-60 \mathrm{~ns}$, are chosen above $t_{g}=180$ and $80 \mathrm{~min}$, where the gelation is completed and the ideal solid network is reached for the corresponding samples. By knowing the $\left[\mathrm{S}_{0}\right]$ value as $9.38 \mathrm{M}, k_{q}$ is measured before the polymerization has started and found to be $4.63 \times 10^{7} \mathrm{M}^{-1} \mathrm{~s}^{-1}$. Using the $k_{q}$ value and the measured $\tau$ values during the gelation process, $[S]$ values are obtained from equation (13). The produced $[S]$ consumption curves versus effective gelation time, $\left(t_{g}-t_{0}\right)$ obey the relation in equation (8). Here the effective gelation time $\left(t_{g}-t_{0}\right)$ is defined as the time at which sol-gel phase transition takes place. The logarithmic form of $[S]$ versus $\left(t_{g}-t_{0}\right)$ are plotted in Figure 10(a), (b) and (c) for samples of 0.025, 0.05 and $0.1 \%$ DVB content where $t_{0}$ is the onset of gelation time which corresponds to $\left[\mathrm{S}_{0}\right]$ value. In Figure 10 the slope of the linear relations produce the composite rate constants, $k_{r}$ according to equation (8), for FCC polymerizations during gelation. The plot of $k_{r}$ versus DVB\% is shown in Figure 11 for all samples. It is observed that, as the DVB content is raised, the composite rate constant increases as expected.

Here it is interesting to plot the maximum $\tau$ values, $\tau_{\max }$, versus DVB concentration in Figure 12 where as DVB is increased $\tau_{\max }$ decrease. This behaviour of $\tau_{\max }$ indicates that more unreacted monomers are trapped in the high DVB content samples than low DVB content samples, after the FCC process is completed. However, after a few days, $\tau_{\max }$ values level off due to polymerization of the leftover monomers. In Figure 12, solid points
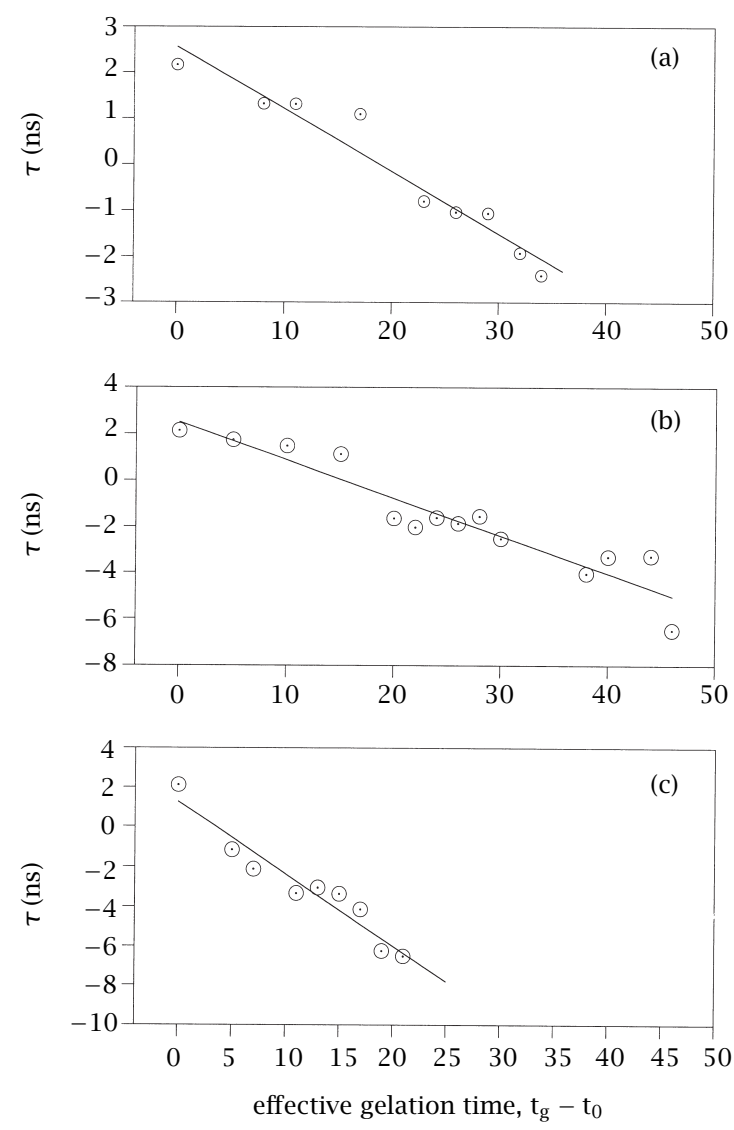

Figure 10. The fit of the data in Figure 8 according to equation (8), where the effective gelation time, $\left(t_{g}-t_{0}\right)$ was used. The slope of the linear relations produce the composite rate constants, $k_{r}$ for the polymerization of $S$ for $a-0.025, b-0.05$ and $\mathrm{c}-0.1 \% \mathrm{DVB}$ content samples.

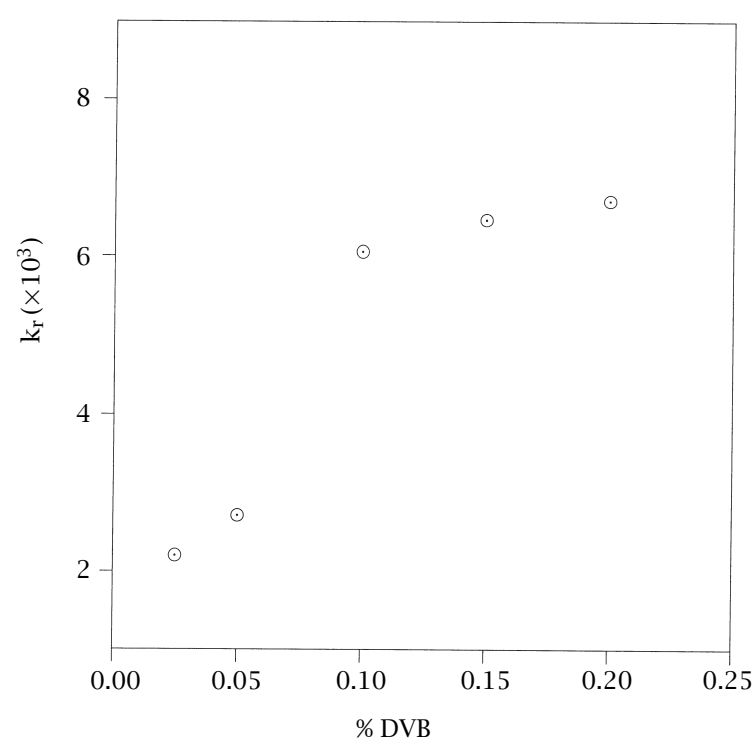

Figure 11. Plot of $k_{r}$ versus crosslinker contents. 


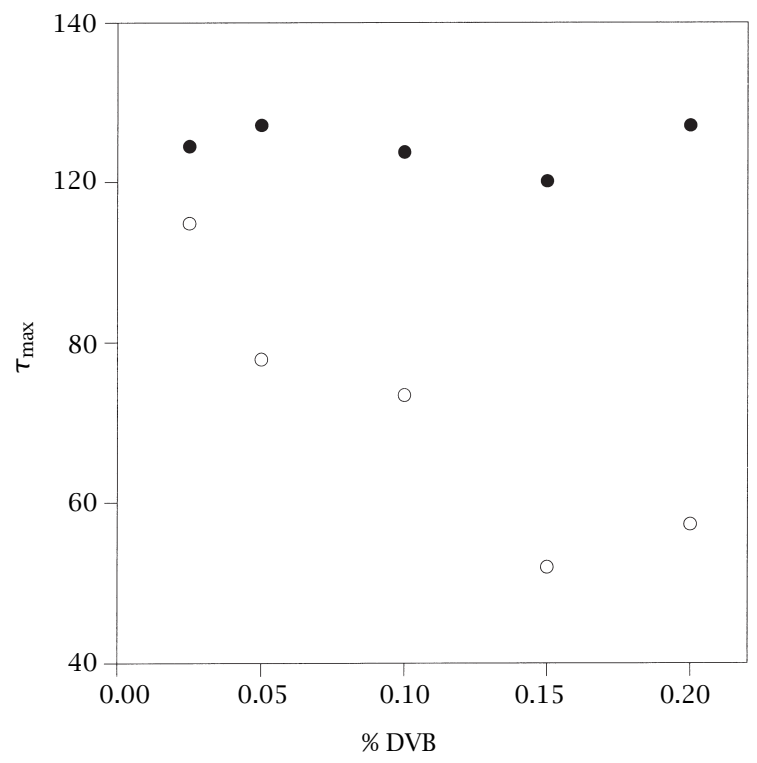

Figure 12. Plot of $\tau_{\max }$ obtained in Figure 8 versus crosslinker contents. Solid points present the $\tau_{\max }$ data a week after the FCC is completed.

represent the $\tau_{\max }$ values measured after a week from the original FCC process. Equation (11) can be used to calculate the amount of unreacted monomer after the FCC process is completed. Here it can be noticed that the similarities between Figure 9 and Figure 12, where it is seen that early and faster polymerization results in having more unreacted monomers trapped in the gel which cause low $\tau_{\max }$ values. However late and slower polymerization results high $\tau_{\max }$ and less unreacted monomers trapped in the gel.

In conclusion, this work has shown that FTRF technique is quite powerful to understand and to study the FCC process during gelation. Measuring fluorescence lifetimes and modelling them can provide us some useful FCC parameters, which are important to be known.

\section{References}

[1] J. B Birks, Photophysics of Aromatic Molecules, J. Wiley-Interscience, New York, 1971.

[2] R. C. Dorrance and T. F. Hunda, J. Chem. Soc. Faraday Trans. 68 (1972), 1312.

[3] S. Cheng and J. K. Thomas, Radiat. Res. 60 (1974), 268.

[4] K. Kalyanasundaram and J. K. Thomas, J. Am. Chem. Soc. 99 (1977), 2039.

[5] Ö. Pekcan, M. A.Winnik, and M. D.Croucher, Macromolecules 16 (1983), 669.

[6] Ö. Pekcan, M. A. Winnik, and M. D. Croucher, J. Polym. Sci. Poly. Lett. 21 (1983), 1011.

[7] Ö. Pekcan, M. A. Winnik, and M. D. Croucher, Phys. Rev. Lett. 61 (1988), 641.
[8] Ö. Pekcan, L. S. Egan, M. A. Winnik, and M. D.Croucher, Macromolecules 23 (1990), 2210.

[9] Ö. Pekcan, Chem. Phys. Lett. 20 (1992), 198.

[10] M. A. Winnik, in Polymer Surfaces and Interfaces, J. Feast and H. Munro (eds.), Wiley, London, 1983, Chapt. 1.

[11] M. A. Winnik, Ö. Pekcan, L. Chen, and M. D. Croucher, Macromolecules 21 (1988), 55.

[12] Ö. Pekcan, J. App. Polym. Sci 59 (1996), 521.

[13] D. Stauffer, Introduction to Percolation Theory, Taylor and Francis, London, 1985.

[14] D. Stauffer, A. Coniglio, and M. Adam, Adv. Polym. Sci. 44 (1982), 103.

[15] H. Herrmann, J. Phys. Rev. 153 (1986), 136.

[16] P. J. Flory, J. Am. Chem. Soc. 63 (1941), 3083.

[17] W. H. Stockmayer, J. Chem. Phys. 11 (1943), 45.

[18] A. G. Mikos, C. G. Takoudis, and N. A. Peppas, Macromolecules 19 (1986), 2174.

[19] H. Tobita and A. E.Hamielec, Macromolecules 22 (1989), 3098.

[20] G. L. Batch and C. W. Macosko, J. Appl. Polym. Sci. 44 (1992), 1711.

[21] O. Okay, Polymer 35 (1994), 796.

[22] K. Dusek, J. Macromol. Sci-Chem. A28 (1991), 843.

[23] J. C. Panxviel, B. Dunn, and J. J. Zink, J. Phys. Chem. 93 (1994), 2134.

[24] Ö. Pekcan, Y. Yýlmaz, and O. Okay, Chem. Phys. Lett. 229 (1994), 537.

[25] Y. Yýlmaz, Ö. Pekcan, and O. Okay, Polymer 37 (1996), 2049.

[26] Ö. Pekcan, Y. Yýlmaz, and O. Okay, J. App. Polym. Sci. 61 (1996), 2279.

[27] Ö. Pekcan and Y. Yýlmaz, Prog. Coll. and Polym. Sci. 102 (1996), 89.

[28] Ö. Pekcan, Y. Yýlmaz, and O. Okay, Polymer 38 (1997), 1693.

[29] Y. Yýlmaz, A. Erzan, and Ö. Pekcan, Phys. Rev. E 58 (1998), 7487.

[30] O. Okay, D. Kaya, and Ö. Pekcan, Polymer (in press).

[31] L. J. Kropp and R. W. Dawson, Fluorescence and Phosphorescence of Aromatic Hydrocarbons in Polymethylmethacrylate, in International Conference on Molecular Luminescence, E. C. Lim (ed.), New York, 1969.

[32] M. Bixon and J. Jortner, J. Chem. Phys. 48 (1968), 715.

[33] J. B. Birks, M. D. Lumb, and I. H. Munro, Proc. Roy. Soc. A, 277 (1964), 289.

[34] K. Kamioka, S. E. Weber, and Y. Morishima, Macromolecules 21 (1988), 972.

[35] P. F. Jones and Siegel, J. Chem. Phys. 50 (1964), 1134.

[36] R. J. Young, Introduction to Polymers, Chapman and Hall, NY., 1983.

[37] W. R. Ware, D. R. James, and A. Siemiarczuk, Rev. Sci. Instrum. 63 (1992), 1710. 


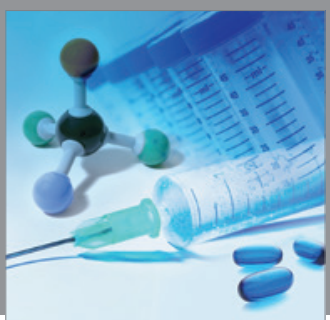

International Journal of

Medicinal Chemistry

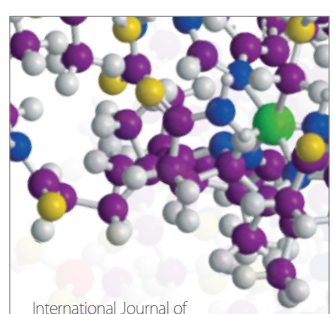

Carbohydrate Chemistry

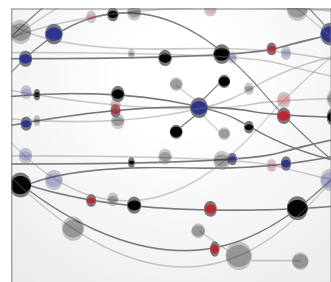

The Scientific World Journal
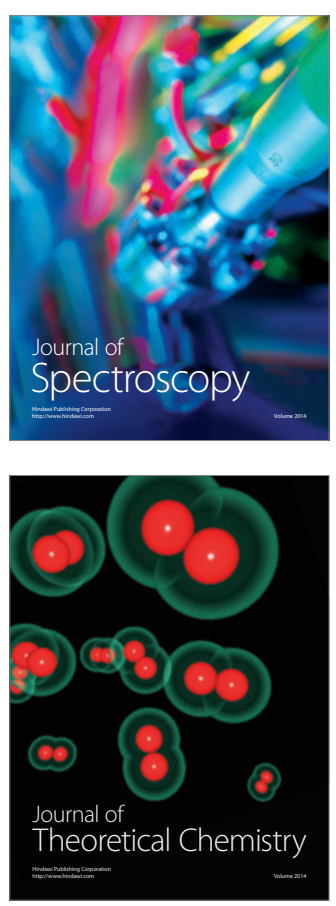
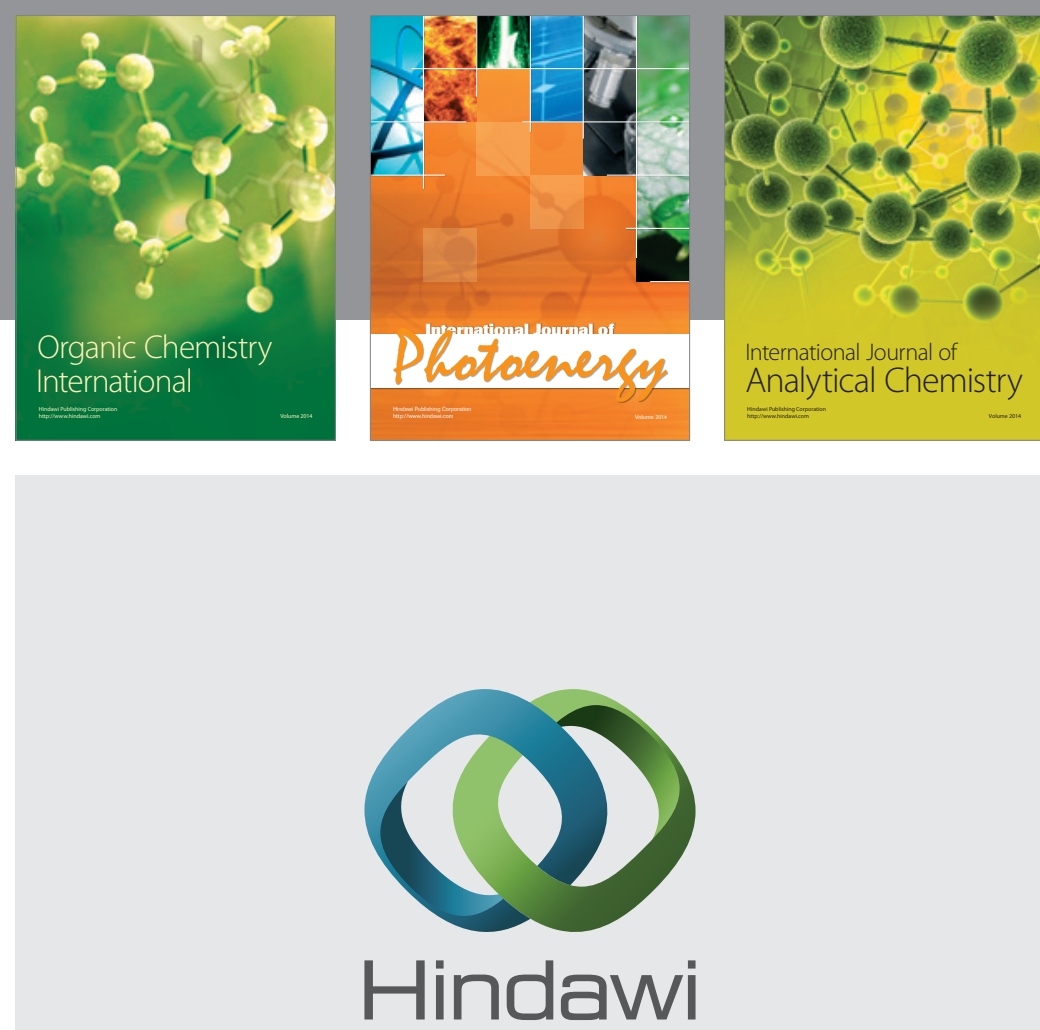

Submit your manuscripts at

http://www.hindawi.com
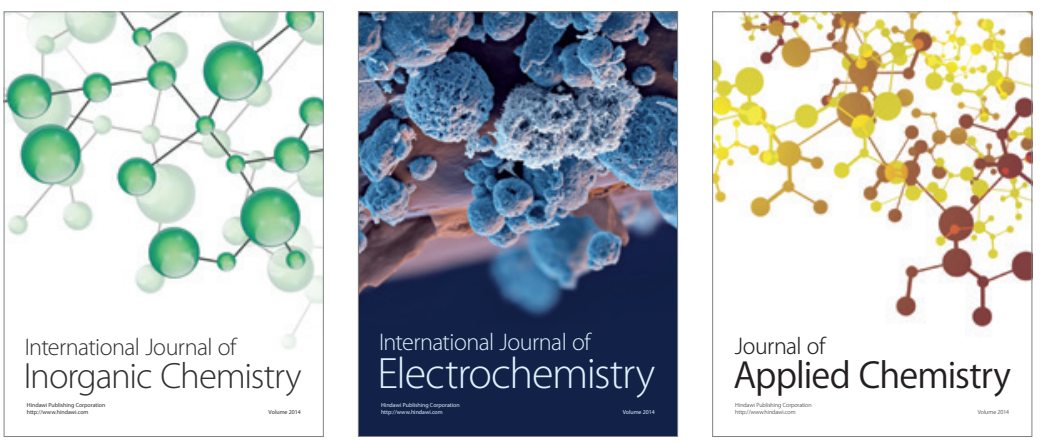

Journal of

Applied Chemistry
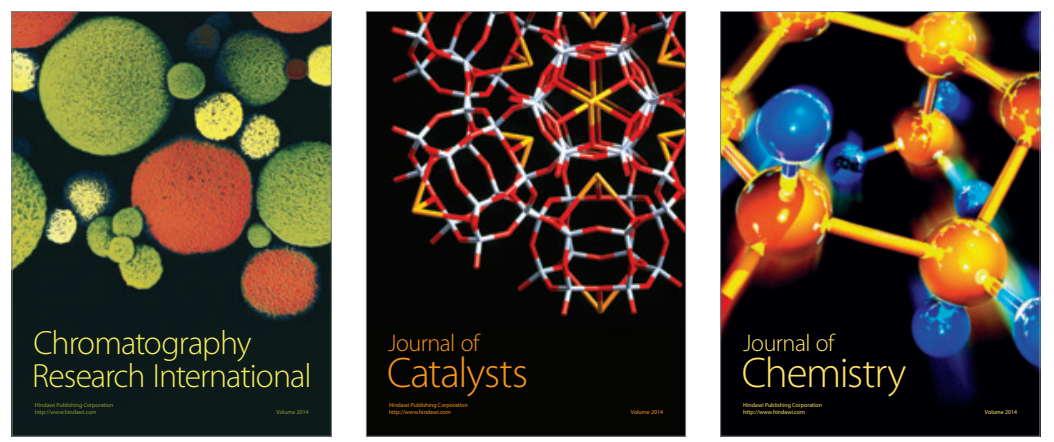
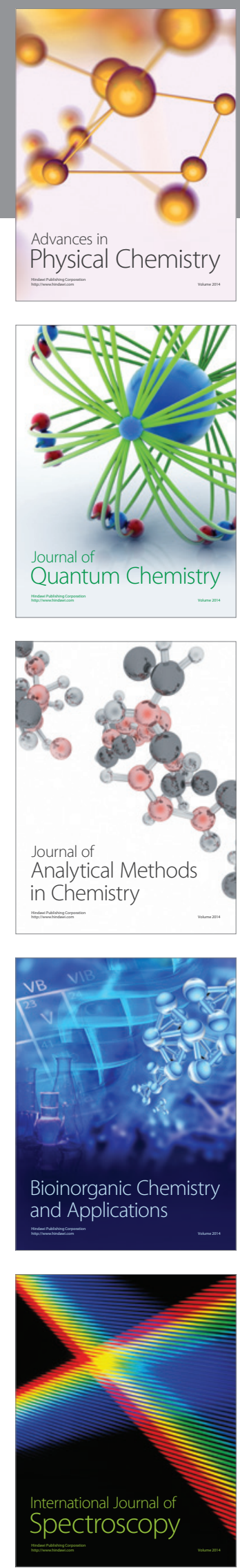